OPEN ACCESS

Edited by: Qiyuan Li,

Xiamen University, China

Reviewed by:

Zhong Wang,

Dalian University of Technology, China

Weifeng Wang,

OrigiMed Inc, China

*Correspondence:

Shun-Dong Dai

daishundong@qq.com

Specialty section:

This article was submitted to Molecular and Cellular Oncology,

a section of the journal

Frontiers in Oncology

Received: 17 January 2021 Accepted: 26 October 2021

Published: 11 November 2021

Citation:

Dai SD, Wang S, Qin YN and Zhu JC (2021) Multiomics Landscape Uncovers the Molecular Mechanism of

the Malignant Evolution of Lung Adenocarcinoma Cells to Chronic Low

Dose Cadmium Exposure.

Front. Oncol. 11:654687. doi: 10.3389/fonc.2021.654687

\section{Multiomics Landscape Uncovers the Molecular Mechanism of the Malignant Evolution of Lung Adenocarcinoma Cells to Chronic Low Dose Cadmium Exposure}

\author{
Shun-Dong Dai ${ }^{1 *}$, Shuang Wang ${ }^{2}$, Ya-Nan Qin ${ }^{1}$ and Jin-Chao Zhu ${ }^{1}$ \\ 1 Department of Pathology, Shanghai Ninth People's Hospital, Shanghai JiaoTong University School of Medicine, \\ Shanghai, China, ${ }^{2}$ Department of Pathology, Shenyang Red Cross Hospital, Shenyang, China
}

Cadmium (Cd) from cigarette smoke and polluted air can lead to lung adenocarcinoma after long-term inhalation. However, most studies are based on short-term exposure to this toxic metal at high concentrations. Here, we investigate the effects of long-term exposure of A549 cells (lung adenocarcinoma) to cadmium at low concentrations using morphological and multiomics analyses. First, we treated A549 cells continuously with $\mathrm{CdCl}_{2}$ at $1 \mu \mathrm{M}$ for 8 months and found that $\mathrm{CdCl}_{2}$ promoted cellular migration and invasion. After that, we applied transmission electron and fluorescence microscopies and did not observe significant morphological changes in Golgi apparatus, endoplasmic reticulum, lysosomes, or mitochondria on Cd treated cells; microfilaments, in contrast, accumulated in lamellipodium and adhesion plaques, which suggested that Cd enhanced cellular activity. Second, by using whole-exome sequencing (WES) we detected 4222 unique SNPs in Cd-treated cells, which included 382 unique non-synonymous mutation sites. The corresponding mutated genes, after GO and KEGG enrichments, were involved mainly in cell adhesion, movement, and metabolic pathways. Third, by RNA-seq analysis, we showed that 1250 genes (784 up and 466 down), 1623 mRNAs (1023 up and 591 down), and 679 IncRNAs (375 up and 304 down) were expressed differently. Furthermore, GO enrichment of these RNA-seq results suggested that most differentially expressed genes were related to cell adhesion and organization of the extracellular matrix in biological process terms; KEGG enrichment revealed that the differentially expressed genes took part in 26 pathways, among which the metabolic pathway was the most significant. These findings could be important for unveiling mechanisms of Cd-related cancers and for developing cancer therapies in the future.

Keywords: cadmium, chronic exposure, lung adenocarcinoma, whole-exome sequencing (WES), RNA sequencing (RNAseq) 


\section{INTRODUCTION}

Cadmium (Cd) is one of the known toxic and carcinogenic transition metals that is distributed widely in the environment. It was classified as a class I human carcinogen by the International Agency for Research on Cancer (IARC) in 1993 (1). The lung is a primary target organ of exposure to cadmium because this metal is mainly absorbed through inhalation $(2,3)$. Cadmium has a very long biological half-life, which result in accumulative toxic and carcinogenic effects (4). Therefore, the harm to the human body also shows long-term and multifaceted characteristics (3). However, most previous studies described the potential of cadmium to cause carcinogenic effects using short-term exposure to this toxic metal at high concentrations $(5,6)$. To mimic conditions more similar to occupational and cadmium exposure that is relevant biologically (7), we exposed human lung adenocarcinoma cell line A549 at a concentration of $1 \mu \mathrm{M}$ for 8 months in our study.

Pulmonary cancer is one of the leading malignant tumors in the world. Despite, the existence of various chemo/physical/ immunological therapies, pulmonary cancer in many countries still has a low 5-year survival rate $(<15 \%)$ (8). Indeed, epidemiological investigations (using specific pollution) and experimental studies (using laboratory animals) have both demonstrated that $\mathrm{Cd}$ exposure increased the risk of pulmonary adenocarcinomas $(3,9)$. However, the specific mechanism of how cadmium exposure promoted the development of lung cancer has not been documented. Carcinogenesis is a multi-stage process that involves a multitude of alterations to the cell. Several studies have focused on how cadmium exposure induced malignant degeneration of bronchial epithelial cells or alveolar epithelial cells, but cadmium promotion of cancer development is involved in all stages of cancer development. There is still a lack of research on how cadmium exposure promotes the malignant progression of lung cancer. In our study, human lung A549 cells were chosen because they display many differentiated features of lung alveolar cells and have been used by many researchers in the cadmium toxicity studies (10-12).

With the advent of next generation sequencing technologies, recent carcinogenic investigations have focused on studying global exome and transcriptome changes to understand the molecular basis of cancer $(13,14)$. Omics data, such as exome, transcriptomics, and epigenomics, provide us an integrated global functional view on cellular responses after exposure to environmental substances. These responses can be linked to the generation and progression of disease. Genome/exome-wide data sets, a complication of large, curated, mechanistic databases, and bioinformatic predictions can deepen our understanding of the sequence of events; these understandings can help us to propose a hypothesis, which can be validated using experimental approaches (15). In this study, we aim to investigate somatic genetic alterations of adenocarcinoma (A549 cells) after longterm, low dose cadmium exposure by using whole-exome sequencing (WES) and RNA-sequencing analyses.

\section{RESULTS}

\section{Effects of Chronic Low Dose Cadmium Exposure on Cytoskeleton, Mitochondria, Endoplasmic Reticulum, Golgi Apparatus, and Lysosomes of A549 Cells}

To determine the influence of cadmium (Cd) on A549 cells, we performed the following experiments: 1) verification of the identity of the A549 cell line; and 2) imaging the cellular ultrastructure and organelle morphology. DNA fingerprinting using short tandem repeat (STR) profiling is an easy and reliable tool that can be used to verify cell lines. It does not change significantly with cell passage number (16). For verification, we confirmed the identity of A459 cells and excluded crosscontamination possibilities in long-term cell culture, by profiling DNA fingerprinting with the STR (Supplementary Figure 1). The results showed that Cd-treated $(\mathrm{A} 549+\mathrm{Cd})$ cells and Cd-untreated $(\mathrm{A} 549+\mathrm{H} 0)$ cells had essentially identical profiles, which indicated no cross-contamination in these two cell lines.

A great deal of evidence suggests that the biphasic nature of cadmium is characterized by low-dose stimulation and high-dose inhibition $(17,18)$. To evaluate this hypothesis in our experimental system, we treated A549 cells with a low concentration $(1 \mu \mathrm{M})$ of cadmium chloride $\left(\mathrm{CdCl}_{2}\right)$ for 8 months. For ultrastructure imaging, we used a transmission electron microscope (TEM) and found that long-term exposure to $\mathrm{CdCl}_{2}$ did not change the ultrastructure of A549 cells noticeably (Figures 1A, B), which suggested Cd did not exhibit a biphasic nature on A549 cells in this setting.

For imaging of organelle morphology, we probed the mitochondria, the endoplasmic reticulum (ER), the Golgi, and lysosomes with fluorescent dyes. No significant morphological change was found in Golgi apparatus, endoplasmic reticulum, lysosomes, or mitochondria after the long-term exposure to $\mathrm{Cd}$ (Figures 1C-F). However, when we probed the actin cytoskeleton, we found that, actin was apparently more disordered after Cd treatment (Figure 1G). The cytoskeleton is a complex of detergent-insoluble components of the cytoplasm that play critical roles in cell motility, shape generation, and mechanical properties of a cell. These results implied that longterm exposure to low doses of $\mathrm{CdCl}_{2}$ might affect organization of A549 cells in the actin cytoskeleton.

\section{Promoting Adenocarcinoma A549 Migration and Invasion}

To examine the effects of chronic low dose $\mathrm{CdCl}_{2}$ on migration and invasion of A549 cells, we performed the Transwell invasion assay and the Scratch test migration assay. Our invasion assay showed that $\mathrm{Cd}$ exposure increased the cell numbers of invasion; the cell numbers of $\mathrm{A} 549+\mathrm{Cd}$ group and $\mathrm{A} 549+\mathrm{H} 0$ group were $144.3 \pm 7.41$ and $98.5 \pm 12.86((\mathrm{t}=4.965, \mathrm{p}=0.0157)$, respectively (Figure 2A). The migration assay showed that A549+Cd cells exhibited increased migration ability compared with A549+H0 cells, as demonstrated by the narrower scratching gap between 
A
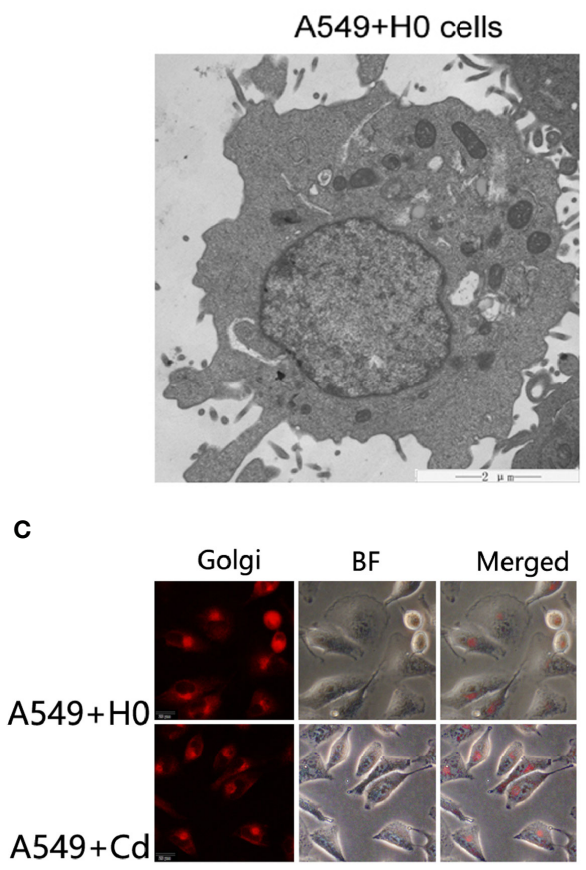

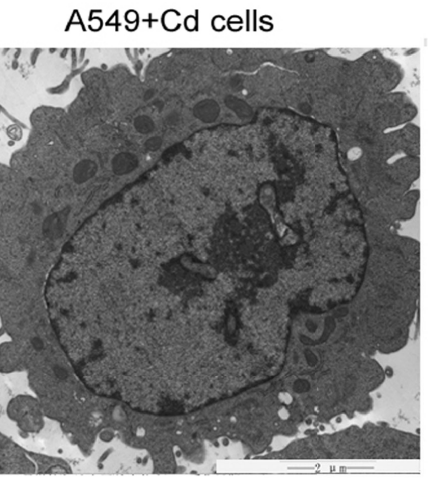

D

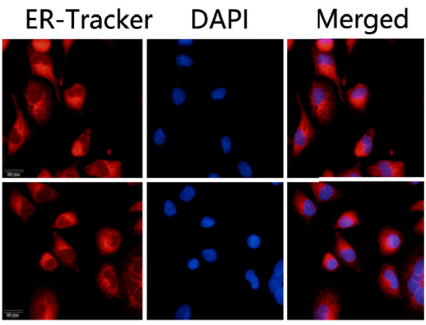

$\mathbf{F}$

E

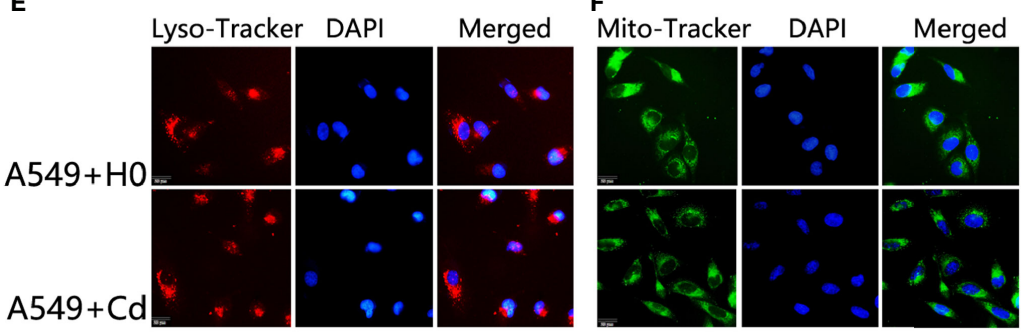

G

Actin-Tracker

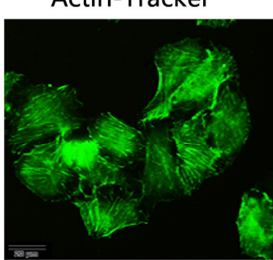

$\mathrm{A} 549+\mathrm{HO}$

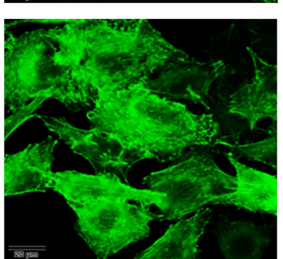

DAPI
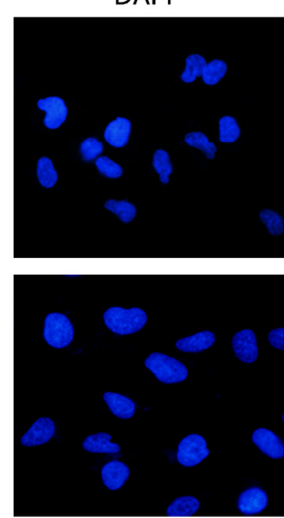

Merged
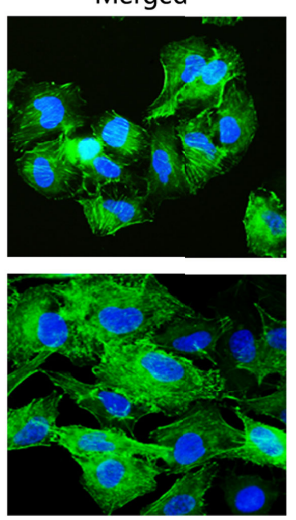

FIGURE 1 | Morphology of cellular organelles and cytoskeleton after Cd exposure. Intracellular structures of A549 cells were imagined by a transmission electron microscope (TEM) after chronic low-dose cadmium (Cd) exposure. (A, B) Ultrastructure comparisons of A549 cells: A549+H0 as blank compared with A549+Cd as Cd treatments. (C-F) No significant morphological changes were found in Golgi apparatus, endoplasmic reticulum (ER), lysosomes (LysoE), or mitochondria (Mito) after Cd treatments. (G) Cytoskeleton disorder was observed in Cd treatment group (A549+Cd). 
A

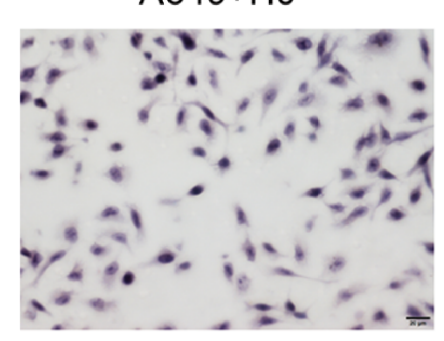

B
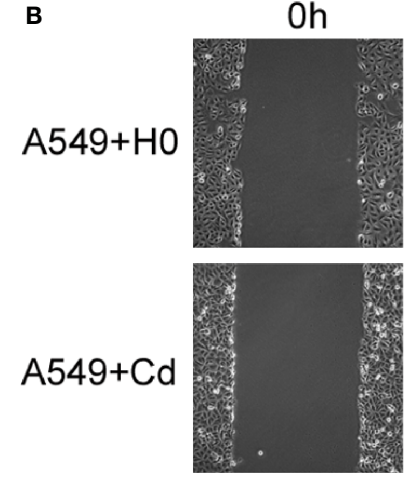

$6 \mathrm{~h}$
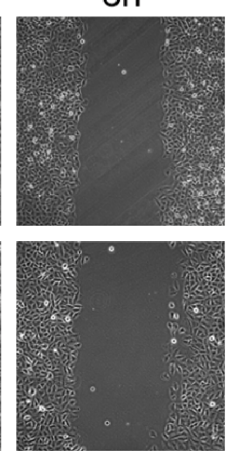

\section{$\mathrm{A} 549+\mathrm{Cd}$}

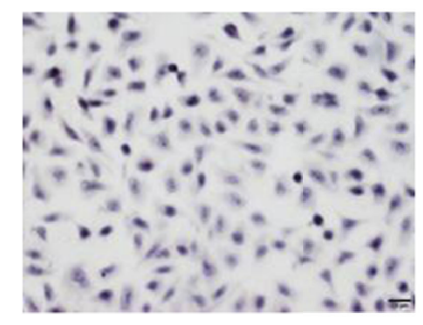

$12 \mathrm{~h}$
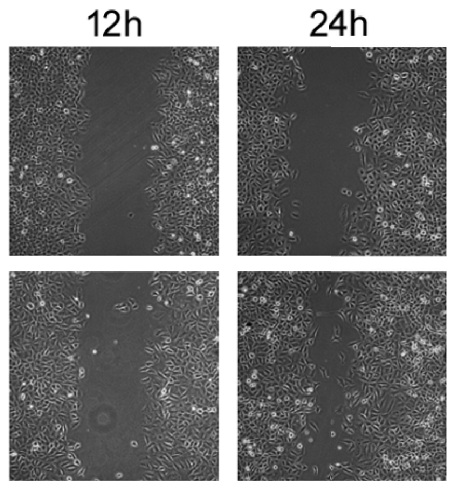

FIGURE 2 | Invasion and migration of A549 cells. (A) Cell invasion was analyzed by the Matrigel Transwell assay. Cd exposure promoted cell invasive behaviors as indicated by more purple cells in the A549+Cd group. (B) Cell migration assays showed narrow gaps in A549+Cd group, which indicated that Cd can promoted the immigration capacity of A549 cells.

cells (Figure 2B). These results indicated that chronic cadmium exposure, even at a low dose, may affect cell invasion and migration.

\section{Genomic Variations in A549 Cells Due to Chronic Low Dose Cadmium Exposure}

As a significant development of next-generation sequencing, WES is a powerful tool for evaluating genomic variation. To identify genomic variation between the A549+Cd cells and A549+H0 cells, we performed high- through put, WES, which generated a total of $\sim 33.0 \mathrm{~Gb}$ of data (>20× depth, $87 \times$ depth max) (Table 1). Based on the sequencing data, we characterized a total of 58,289 SNPs in A549+Cd cells and 50,887 SNPs in A549+H0 cells (Figure 3 and Supplemental Data 1).

For further biological analyses, we selected 6876 nonsynonymous mutation SNP sites, 125 non-frameshift mutations, 64 frameshift mutations, 52 terminator acquisition loci, and six terminator loss loci from both cells. Subsequently, 382 unique nonsynonymous mutation SNPs were detected in the A549+Cd cells (Supplemental Data 2) compared with A549+H0 cells. Among these 382 SNPs, 78 affected genes were associated with cell migration and invasion ability. However, none of them harbored a specific pathogenic mutation based on rigorous evaluation (Pathogenicity Calculator, http://calculator.clinicalgenome.org/site/ cg-calculator) (Supplemental Data 3). To explore the affected 78 genes in human lung adenocarcinoma, we downloaded human lung adenocarcinoma SNP mutation data and transcriptome data (normal: 54, tumor: 497) (TCGA-LUAD) from a TCGA database.
We found that the mutation rates of TTN, PCLO, RYR3, and LRP2 in human lung adenocarcinoma were $41 \%, 16 \%, 14 \%$ and $11 \%$, respectively, but the mutation rates of other mutant genes were $<10 \%$ (Figure 4A and Table 2). From the DESeq differential analysis, 1826 up-regulated genes and 1295 down-regulated genes were obtained from transcriptome date of TCGA lung adenocarcinoma. By taking the intersection as shown in Figure 4B, the expressions of CKAP2L, FAT1, GSDMC, MUC4, KRT15, KIF18B and SKA1 of 78 affected genes were up-regulated in the TCGA database, MYH11, SPTBN1, LAMC3 and CD33 were down-regulated, and other genes were not expressed differentially in lung adenocarcinoma of TCGA.

Functional annotation analysis of genes in the associated regions was performed using different databases, which included NCBI non-redundant (NR), The Gene Ontology (GO), Clusters of Orthologous Groups (COG), and Kyoto Encyclopedia of Genes and Genomes (KEGG) Pathway analysis. According to GO enrichment analysis, the unique mutant genes were enriched in $30 \mathrm{GO}$ terms, which included 10 biological process terms (BP), 10 molecular function terms (MF), and 10 cellular component terms (CC). In BP terms, most of the mutated gene enrichments, involved adhesion and cell movement, such as biological adhesion, cell adhesion, homophilic cell adhesion, and actin filament-based movement (Table 3 and Figure 5). In MF terms, mutated genes were largely related to metal ion binding, cation binding, and calcium ion binding. In CC terms, major mutations were involved in extracellular regions and the cytoskeleton. 
TABLE 1 | Exon capture statistic of A549+H0 cells and A549+Cd cells.

Exon Capture Statistics

Target size(bp)

Effective reads

Aligned reads

$\%$ Aligned reads

Reads in target region

$\%$ Reads in target region

Mean depth of target region

Coverage of target region

Reads in flanking region

$\%$ Reads in flanking region

Mean depth of flanking region

Coverage of flanking region

$\%$ Targeted region covered at depths of at least $4 \mathrm{X}$

$\%$ Targeted region covered at depths of at least $10 \mathrm{X}$

$\%$ Targeted region covered at depths of at least $20 \mathrm{X}$

$\%$ Flanking region covered at depths of at least $4 \mathrm{X}$

$\%$ Flanking region covered at depths of at least $10 \mathrm{X}$

\%Flanking region covered at depths of at least $20 \mathrm{X}$

Non-duplicated reads

$\%$ Non-duplicated reads

Uniq mapped reads

\%Uniq mapped reads
A549+H0 cells DNA

A549+Cd cells DNA

$$
\begin{gathered}
62,085,295 \\
69,157,446 \\
67,779,635 \\
98.01 \\
34,499,846 \\
50.90 \\
83.35 \\
97.9247275865 \\
24,521,219 \\
36.18 \\
51.18 \\
95.67 \\
97.81 \\
97.38 \\
96.25 \\
94.92 \\
92.00 \\
84.22 \\
55,532,142 \\
80.30 \\
48,971,224 \\
70.81
\end{gathered}
$$

$$
\begin{gathered}
62,085,295 \\
57,625,498 \\
56,391,115 \\
97.86 \\
29,664,782 \\
52.61 \\
71.67 \\
97.5160655998 \\
20,989,258 \\
37.22 \\
43.81 \\
93.39 \\
97.38 \\
96.75 \\
94.94 \\
92.37 \\
88.11 \\
76.81 \\
47,132,701 \\
81.79 \\
41,493,173 \\
72.00
\end{gathered}
$$

(four mutated genes), glycosaminoglycan degradation (two mutated genes), drug metabolism-cytochrome P450 (five mutated genes), and chemical carcinogenesis (five mutated genes) (Figure 5B).

\section{Profiles of Transcriptomes Due to Chronic Low Dose Cadmium Exposure of A549 Cells to Cadmium}

To characterize transcriptomic differences after long-term exposure to $\mathrm{Cd}$, we conducted next-generation RNA metabolism genes (three mutated genes), carbon metabolism

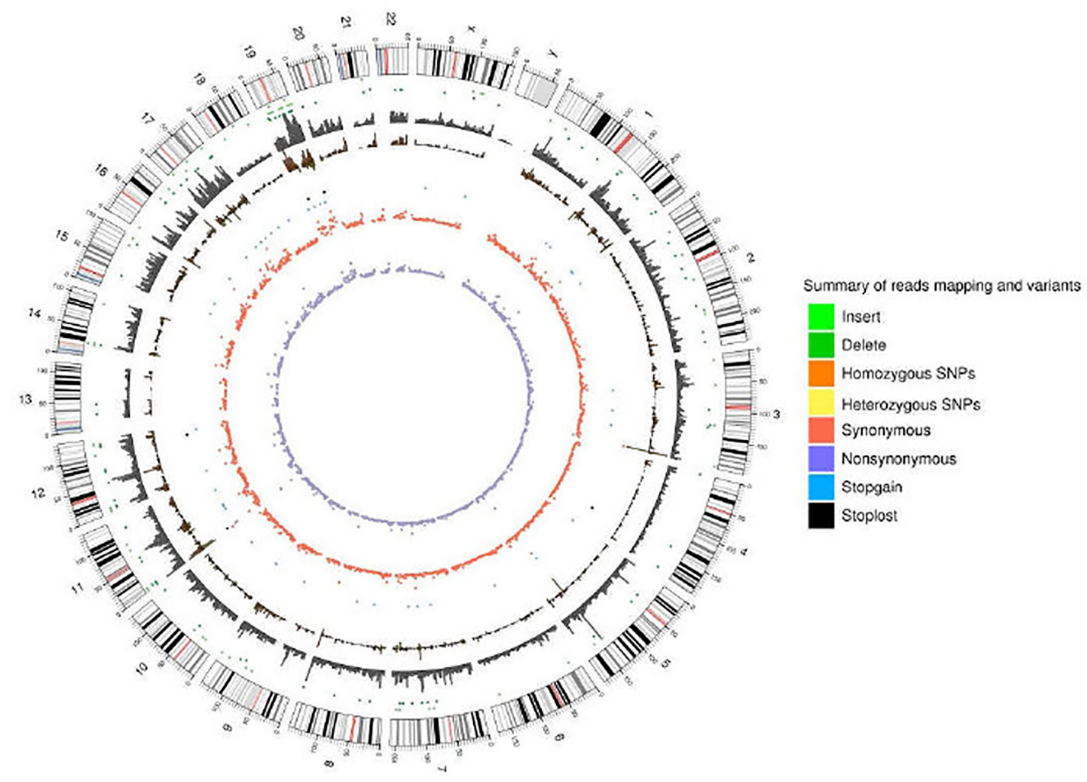

FIGURE 3 | Reads coverage of each chromosome and the statistical results of variation loci. 
A

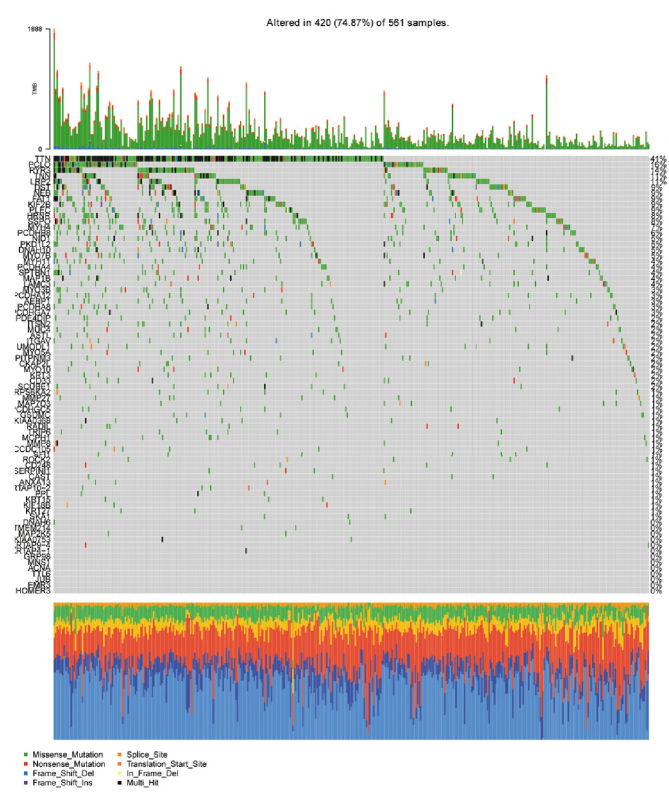

B

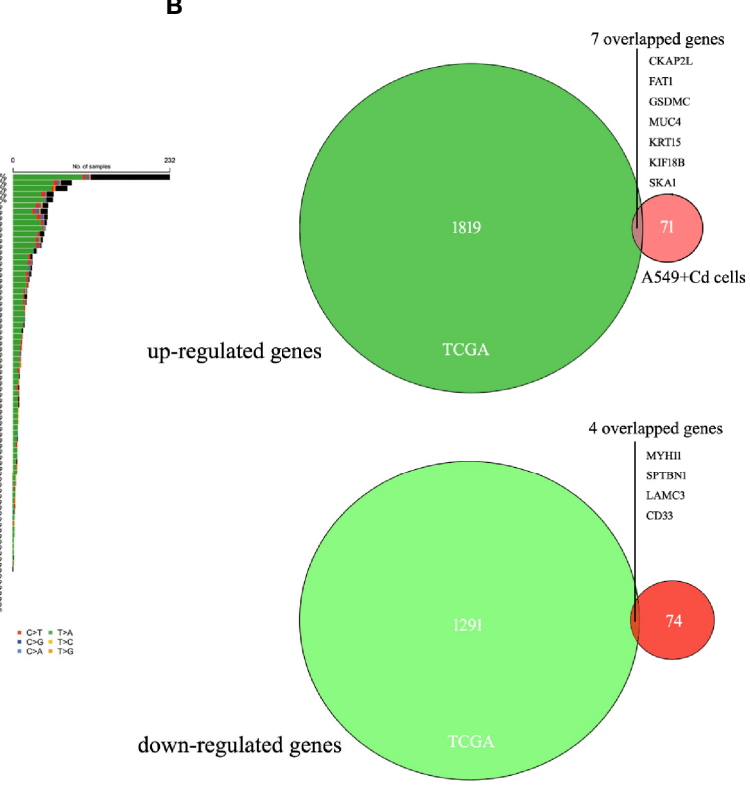

FIGURE 4 | Seventy-eight mutated genes associated with cell migration and invasion were identified in the TCGA lung adenocarcinoma database. (A) Waterfall of 78 mutant genes in TCGA lung adenocarcinoma. The most frequently mutated genes were TTN (41\%), PCLO (16\%), RYR3(14\%) and LRP2 (11\%). (B) Venn diagrams of overlapping up-regulated genes and down-regulated genes in TCGA database and A549+Cd cells.

sequencing. Two groups of cells were sequenced: 1) A549+Cd cells with 51,201 transcripts $(32,223$ mRNAs and 18,979 lncRNAs); and 2) A549+H0 cells with 51,136 transcripts (31,925 mRNAs and 19,211 lncRNA) (Supplementary Data 4).

By comparing the RNA sequencing data of $\mathrm{A} 549+\mathrm{Cd}$ and A $549+\mathrm{H} 0$ cells, we obtained the differential profile transcriptomes that were caused by chronic, low dose, cadmium exposure. We identified 1250 differentially expressed genes, among which 784 genes were significantly up-regulated and 466 genes were down-regulated in A549+Cd cells, compared with A549+H0 cells. Among them, 132 differentially expressed genes were related to migration; 85 up-regulated genes (Table 4) and 47 down-regulated genes (Table 5). We also identified differentially expressed mRNAs, which included 1023 increased and point 591 decreased mRNAs. Transcriptional data also indicated that there were 679 differentially expressed lncRNAs (375 increased and 304 decreased).

Functional enrichment analysis based on GO annotations showed that the differentially expressed genes of A549+Cd cells were enriched in $30 \mathrm{GO}$ terms, which included 10 biological process (BP) terms, 10 molecular function (MF) terms, and 10 cellular component (CC) terms (Supplementary Data 5 and Figure 5). Most differentially expressed genes that were enriched in BP terms may be related to cell adhesion and extracellular matrix organization, such as biological adhesion, cell adhesion, which was similar to the GO annotations based on WES. Most differentially expressed genes enriched in MF terms were related to receptor binding and protein heterodimerization activity. In addition, most differentially expressed genes enriched in CC terms were related to extracellular regions and integral/intrinsic components of plasma membrane. These results from the transcriptomes were similar to the results of the entire exome, which indicated that $\mathrm{Cd}$ long-term exposure may affect development of carcinoma.

Apart from GO annotations, we also performed KEGG enrichment analysis and found that the DEGs were involved in 26 pathways (Supplementary Data 6); the metabolic pathway was the most significant (Figure 6). Other significant pathways

TABLE 2 | Overlapping mutation genes in A549+Cd cells and in the TCGA lung adenocarcinoma database.

\begin{tabular}{|c|c|c|c|c|c|c|c|}
\hline Up-regulated & p-value & q-value & SNP number & Down-regulated & p-value & q-value & SNP number \\
\hline CKAP2L & 4.26E-12 & 9.89E-11 & 10 & $\mathrm{MYH11}$ & $4.52 \mathrm{E}-21$ & 3.02E-19 & 42 \\
\hline FAT1 & 3.37E-05 & 0.000244209 & 58 & SPTBN1 & $9.62 \mathrm{E}-18$ & $4.64 \mathrm{E}-16$ & 27 \\
\hline GSDMC & 2.76E-09 & 4.26E-08 & 10 & LAMC3 & $6.34 \mathrm{E}-10$ & 1.08E-08 & 33 \\
\hline MUC4 & $9.01 \mathrm{E}-11$ & 1.76E-09 & 16 & CD33 & 0.001136 & 0.00566129 & 16 \\
\hline KRT15 & 0.010675 & 0.038861457 & 6 & & & & \\
\hline KIF18B & $1.59 \mathrm{E}-16$ & 6.50E-15 & 4 & & & & \\
\hline SKA1 & $2.63 E-10$ & 4.75E-09 & 3 & & & & \\
\hline
\end{tabular}


TABLE 3 | Gene Ontology(GO) enrichment analysis of mutated genes in A549+Cd cells.

\begin{tabular}{|c|c|c|}
\hline GOMFID & Term & Gene Names \\
\hline GO:0005516 & $\begin{array}{l}\text { calmodulin } \\
\text { binding }\end{array}$ & TTN;RYR3;OBSCN;MYO7A;MYO5A;MYH4;MYO10;MYH7;AEBP1;MYH11;SPTBN1 \\
\hline GO:0008237 & $\begin{array}{l}\text { metallopeptidase } \\
\text { activity }\end{array}$ & ADAMTS7;MMP8;ADAMTS17;ERAP1;DNPEP;MMP27;ADAMTSL3;ADAMTS1;CPB2;ASTL;AEBP1;CPA4 \\
\hline GO:0000146 & $\begin{array}{l}\text { microfilament } \\
\text { motor activity }\end{array}$ & MYO3B;MYO7A;MYO5A;MYH4;MYO10;MYH7 \\
\hline $\begin{array}{l}\text { GO:0003774 } \\
\text { Cellular Compo }\end{array}$ & $\begin{array}{l}\text { motor activity } \\
\text { onent }\end{array}$ & KIF2B;DNAH6;MYO7B;MYO3B;MYO7A;MYO5A;MYH4;MYO10;MYH7;DNAH10;MYH11;KIF18B \\
\hline GO:0005576 & $\begin{array}{l}\text { extracellular } \\
\text { region }\end{array}$ & $\begin{array}{l}\text { PKD1;ADAMTS7;IMPG1;DST;ANXA13;ACAN;KRT15;ALDH3A1;PSMB1;TPSAB1;MMP27;FAT1;ZNF559-ZNF177;PLEC;EMR3; } \\
\text { UPK3A;CUBN;PPL;SERPIN1;:WDR60;NEB;BTBD17;ACR;SLC1A5;ADAMTS17;C17orf99;SLC12A3;ERAP1;PODN;A2M;CLEC18C; } \\
\text { GPR98;RSPO2;TIAM2;ZNF177;ZNF446;CPA4;ADAMTS1;CD248;LAMC3;WWA8;KLK1;MMP8;INHA;ITGAV;FABP1;CCDC147; } \\
\text { DNPEP;ITSN2;ADAMTSL3;HRNR;AP4M1;LRP2;APOA1BP;AEBP1;PNP;GSTP1;UMODL1;SPTBN1;TTN;NID1;CHIA;VASN; } \\
\text { RAB3GAP1;KLK13;SORL1;TPSB2;PGC;EPN3;OTOP1;TNN;ENO3;MUC5B;MUC6;CPB2;MUC4;JUP;SSPO;CCDC105;SCUBE1; } \\
\text { MUC2;SLC9A3 }\end{array}$ \\
\hline GO:0016459 & myosin complex & MYO7B;MYO3B;MYO7A;MYO5A;MYH4;MYO10;MYH7;MYH11 \\
\hline GO:0044430 & cytoskeletal part & $\begin{array}{l}\text { KIF2B;MNS1;GSDMC;CCDC105;DST;TTLL6;KRT15;ROCK2;KIAA0753;MAP2K5;MYO10;DNAH10;PDE4DIP;RADIL;KIAA0368;SFI1; } \\
\text { MYO3B;DNAH6;KRTAP9-4;HOMER3;KRTAP4-1;KRT3;KIF18B;MYO7A;MAP7D3;MCPH1;MYH4;JUP;MYH7;MYH11;CKAP2L; } \\
\text { SPTBN1;TTN;MYO7B;KRTAP10-2;TMEM214;SKA1;KRT27;ITPR1;MYO5A;RPS6KA2;MAP1B;EML1 }\end{array}$ \\
\hline \multicolumn{3}{|c|}{ Biological Process } \\
\hline GO:0007156 & $\begin{array}{l}\text { homophilic cell } \\
\text { adhesion }\end{array}$ & PKD1;PCDHA8;PCDHA4;FAT1;PCDHGA7;PCDHB8;PCDHGC5;PCDH8;PCDHB12 \\
\hline GO:0030048 & $\begin{array}{l}\text { actin filament- } \\
\text { based movement }\end{array}$ & TTN;NEB;MYO7A;MYO5A;MYH4;MYH7 \\
\hline GO:0007155 & cell adhesion & $\begin{array}{l}\text { DST;PCDHA8;PCDHA4;MAP2K5;MYO10;FAT1;ACAN;RADIL;SERPIN11;PKD1;TRIP6;GPR98;LAMC3;UUP;ASTL;AEBP1;PCDH8; } \\
\text { ROCK2;PCDHGC5;NID1;CD33;ITGAV;TNN;PCDHB12;PCDHGA7;MUC4;PCDHB8;SSPO }\end{array}$ \\
\hline
\end{tabular}

were chemical carcinogenesis, transcriptional mis-regulation in cancer, cytokine-cytokine receptor interaction, calcium signal pathway, Rap1 signal pathway and Hedgehog pathway.

\section{DISCUSSION}

Cadmium (Cd) is a heavy element that is known to accumulate in the body and to have strong biological effects. The main entry for cadmium into the body include the respiratory tract, digestive tract, and skin contact. As a non-essential element with 20-30 years half-life in vivo, the $\mathrm{Cd}$ can remain in the human kidney and liver for almost a lifetime. Therefore, the harm to the human body also shows long-term and multifaceted characteristics. Because the relationship between cadmium and "pain disease" was put forward in the 1960s, there have been many studies on the relationship between environmental pollution and human health due to cadmium. Due to daily-life and industrial activities, 4000 to 13000 tons of $\mathrm{Cd}$ has been discharged into the environment every year (19). These toxic pollutants can then accumulate in the human body through direct inhalation of air, drinking water, or by eating crops that grew in polluted soil (19).
In addition, occupational exposure and smoking are also important avenues (20). Unlike the kidney and liver, the lung is a primary target organ, directly exposed to Cd pollutants, especially for those people with occupational exposure or for those who smoke tobacco. The affected lung can suffer acute inflammation, chronic edema, bronchitis, and even cancers (21). For the underlying molecular mechanisms of these diseases, $\mathrm{Cd}$ may be a strong inducer for deletion of multiple loci in the genes (or gene mutations). Because it cannot be bound to DNA stably, $\mathrm{Cd}$ might lead to gene mutations by indirect genotoxic mechanisms, such as oxidative stress, inhibition of DNA repair, stimulation of cell proliferation, blockage of apoptosis, and epigenetic mechanisms $(4,22)$.

Although $\mathrm{Cd}$ can induce gene mutation indirectly and promote the development of cancer, a global picture of the progression of adenocarcinoma after long-term exposure to low-dose Cd is still vague. Previous studies have reported that cadmium affected the biological behavior of cells through Wnt $(23,24)$, PI3K/AKT $(25,26)$ or JNK $(5,27)$ signaling pathways, but there is still a lack of studies on the effect of long-term, low dose cadmium exposure on whole exome and transcriptomes of lung cancer cells in the lung. Cadmium itself has the ability to 
TABLE 4 | Differentially up-regulated genes associated with cell migration and invasion in A549+Cd cells.

\begin{tabular}{|c|c|c|c|c|c|}
\hline Gene & log2FoldChange & q-value & Gene & log2FoldChange & q-value \\
\hline JAG2 & 1.20 & $6.47 \mathrm{E}-15$ & PCDHA3 & 1.17 & $6.47 \mathrm{E}-15$ \\
\hline ANXA13 & 1.36 & 0.000233 & MMP17 & 2.31 & $5.10 \mathrm{E}-15$ \\
\hline FBLN5 & 2.40 & $5.10 \mathrm{E}-15$ & DGKA & 1.71 & 1.11E-09 \\
\hline TNC & 1.33 & $5.10 \mathrm{E}-15$ & CALB2 & 1.14 & $5.72 \mathrm{E}-08$ \\
\hline TLL1 & 1.43 & $1.72 \mathrm{E}-11$ & TLL2 & 1.31 & 0.000299 \\
\hline NCALD & 4.03 & $6.47 \mathrm{E}-15$ & THBS1 & 1.03 & $6.47 \mathrm{E}-15$ \\
\hline SCUBE2 & 1.87 & 1.64E-05 & $\mathrm{PCDH} 20$ & 2.62 & 2.76E-06 \\
\hline EMR1 & 1.02 & $5.10 \mathrm{E}-15$ & RASGRP4 & 1.14 & 4.90E-05 \\
\hline PCDHGA2 & 1.05 & $9.09 E-13$ & $\mathrm{RCN} 3$ & 1.05 & 3.59E-08 \\
\hline PCDHAC2 & 1.10 & $6.47 \mathrm{E}-15$ & $\mathrm{CDH} 22$ & 3.23 & 6.83E-06 \\
\hline C1S & 1.14 & 8.99E-15 & C1R & 1.63 & $8.99 E-15$ \\
\hline PADI3 & 2.45 & $5.10 \mathrm{E}-15$ & PCDHGB3 & 1.11 & $5.10 \mathrm{E}-15$ \\
\hline PCDHA4 & 1.06 & $6.47 \mathrm{E}-15$ & SPARC & 3.02 & $5.10 \mathrm{E}-15$ \\
\hline NPNT & 3.02 & $9.74 \mathrm{E}-08$ & PCDHA10 & 1.00 & $5.10 \mathrm{E}-15$ \\
\hline DSC3 & 2.55 & $6.47 \mathrm{E}-15$ & SPOCK2 & 1.32 & 9.09E-05 \\
\hline PROCA1 & 1.02 & $4.55 \mathrm{E}-10$ & $\mathrm{CDH} 4$ & 1.25 & $6.47 \mathrm{E}-15$ \\
\hline $\mathrm{PCDH} 7$ & 1.01 & 0 & NPHS1 & 1.27 & $6.14 \mathrm{E}-07$ \\
\hline REPS2 & 1.12 & 8.99E-15 & ELTD1 & 4.46 & 9.09E-06 \\
\hline PRRG4 & 1.20 & 5.10E-15 & CDK5R1 & 1.29 & $7.81 \mathrm{E}-11$ \\
\hline PCDHB5 & 2.41 & $5.10 \mathrm{E}-15$ & DLL1 & 1.02 & $6.75 \mathrm{E}-07$ \\
\hline CLDN2 & 1.50 & $5.10 \mathrm{E}-15$ & VAV3 & 1.12 & $6.47 \mathrm{E}-15$ \\
\hline RND1 & 1.73 & $1.59 \mathrm{E}-11$ & $\mathrm{CDH} 16$ & 1.87 & 0 \\
\hline THEMIS2 & 1.35 & 1.03E-07 & ANGPT1 & 1.74 & 1.70E-06 \\
\hline FES & 1.46 & 0.000669 & DSCAML1 & 2.65 & $5.10 \mathrm{E}-15$ \\
\hline NRARP & 1.03 & $1.95 \mathrm{E}-10$ & CLDN3 & 4.06 & $5.10 \mathrm{E}-15$ \\
\hline CD4 & 3.57 & 1.94E-05 & EMB & 2.31 & $6.47 \mathrm{E}-15$ \\
\hline HMCN1 & 1.64 & 0 & NOTCH3 & 2.65 & 0 \\
\hline LRRN2 & 2.55 & 0 & AMIGO1 & 1.17 & 1.18E-09 \\
\hline PDGFRA & 1.31 & 5.66E-05 & TNFAIP6 & 1.79 & 1.15E-05 \\
\hline ITGA4 & 2.58 & $6.47 \mathrm{E}-15$ & ICAM1 & 1.26 & 0 \\
\hline CADM2 & 4.72 & 5.21E-07 & ESAM & 2.40 & $5.59 E-13$ \\
\hline COL1A1 & 1.06 & 4.32E-14 & EPHA7 & 4.91 & $6.47 \mathrm{E}-15$ \\
\hline NEO1 & 1.07 & $6.47 \mathrm{E}-15$ & AMBP & 1.58 & $5.10 \mathrm{E}-15$ \\
\hline SAA1 & 3.53 & $5.10 \mathrm{E}-15$ & ITGB6 & 2.19 & 0 \\
\hline PTPRD & 1.24 & 0 & C1QTNF1 & 3.15 & 0 \\
\hline AZU1 & 1.38 & 7.55E-05 & SERPINF2 & 2.76 & $6.47 \mathrm{E}-15$ \\
\hline HOXA7 & 1.40 & 0.000593 & CCL5 & 3.06 & $5.10 \mathrm{E}-15$ \\
\hline ITGAX & 1.91 & $1.93 \mathrm{E}-10$ & TRO & 2.78 & $5.10 \mathrm{E}-15$ \\
\hline BCAM & 1.41 & $6.47 \mathrm{E}-15$ & EDA & 1.94 & $7.64 \mathrm{E}-13$ \\
\hline KANK1 & 1.18 & $8.22 \mathrm{E}-15$ & MCAM & 1.35 & 0 \\
\hline NTN1 & 1.21 & 1.39E-08 & LAMC2 & 2.18 & $6.47 \mathrm{E}-15$ \\
\hline STAT5A & 1.22 & 5.47E-07 & GREM1 & 3.21 & 1.71E-07 \\
\hline NLGN4Y & 2.23 & $5.10 \mathrm{E}-15$ & & & \\
\hline
\end{tabular}

induce gene mutation indirectly (28) and, therefore, it inhibits the DNA repair system, which results in gene damage and accumulation of mutation. To provide a clear picture, we sequenced whole exome and transcriptomes of A549 cells after a low dose, long-term treatment with $\mathrm{Cd}$ and then analyzed the function of the mutated genes by GO and KEGG enrichments. Our exome results showed that 78 mutated genes were related to biological processes of cell movement and migration, such as calmodulin binding, microfilament movement function, calcium binding function, cytoskeleton composition, actin-related biological processes, and cell adhesion. Our transcriptomic results showed that $\mathrm{Cd}$ affected the expression of genes related to calcium binding, cell adhesion, DNA, and protein synthesis. These influences are signs of transcriptomic disorders in tumor progression. The transcriptomic disorders may lead further to cytoskeleton disorders in A549 cells and then affect biological functions, such as adhesion and migration. Because $\mathrm{Cd}$ was shown previously to regulate Wnt, PI3K/AKT and JNK signaling pathways, we speculated that these pathways together with the disorders found in our studies rendered progression of adenocarcinoma more malignant. Other tumor-related pathways (e.g., Hedgehog pathway, Rap1 signal pathway, and calcium signal pathway, and chemical carcinogenesis) might also crosstalk with those disorders.

Reactive oxygen species (ROS) can be induced by Cd-related cellular disorders, such as decreasing the activity of antioxidant enzymes, imbalance of intracellular calcium homeostasis, interference with cellular calcium metabolism, and damage of the DNA repair mechanism $(29,30)$. Over the last decade, many investigators have highlighted the involvement of ROSstimulated signaling in metal-induced carcinogenesis. Unlike general concepts on the relationship between metal and ROS, a direct role of ROS is not considered likely in cadmium-induced carcinogenesis because cadmium does not participate in Fenton- 
A

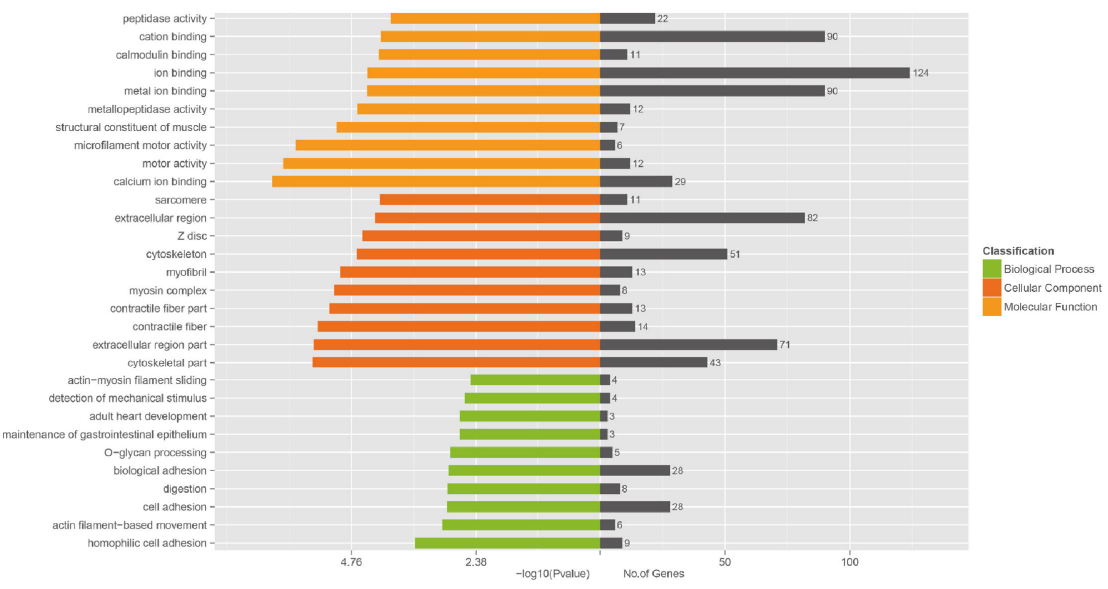

B

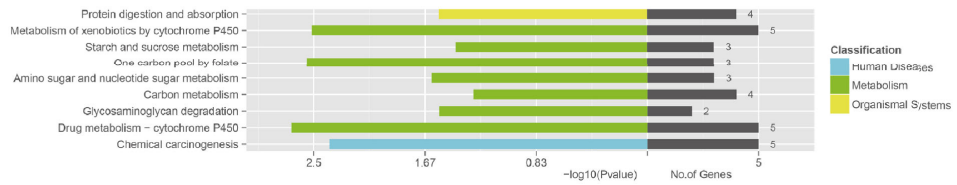

FIGURE 5 | Functional enrichment of unique mutated genes. (A) The Gene Ontology (GO) enrichment of unique mutated genes in A549+Cd cells. GO analysis comprises three branches: cellular components, molecular functions, and biological processes. (B) The Kyoto Encyclopedia of Genes and Genomes (KEGG) pathway analysis of unique mutated genes in A549+Cd cells. KEGG analysis includes three branches: human diseases, metabolisms, and organismal systems.

TABLE 5 | Differentially down-regulated genes associated with cell migration and invasion ability in A549+Cd cells.

\begin{tabular}{|c|c|c|c|c|c|}
\hline Gene & log2FoldChange & q-value & Gene & log2FoldChange & q-value \\
\hline EFCAB12 & -1.54 & 0.000499 & SVEP1 & -1.71 & 1.25E-99 \\
\hline EFEMP1 & -4.82 & 1.19E-304 & EDIL3 & -2.24 & 0 \\
\hline PC & -1.18 & 1.78E-90 & MMP16 & -1.31 & 5.65E-28 \\
\hline FAT3 & -1.31 & $3.66 \mathrm{E}-113$ & CD248 & -2.58 & 0.000567 \\
\hline $\mathrm{CDH} 2$ & -4.77 & 0 & CCBE1 & -3.48 & 5.59E-33 \\
\hline CYP1B1 & -3.19 & 0 & COL12A1 & -2.11 & 1.27E-234 \\
\hline CYR61 & -1.49 & $1.19 \mathrm{E}-174$ & ICAM5 & -1.18 & 2.86E-07 \\
\hline HAS2 & -1.88 & 1.80E-06 & MSLN & -2.06 & 0.000122 \\
\hline FOXC2 & -3.18 & 0.000930 & VEGFA & -1.17 & 7.98E-303 \\
\hline HAPLN2 & -1.04 & 0.000876 & TENM3 & -1.39 & 4.13E-209 \\
\hline NLGN1 & -1.35 & 1.06E-25 & ITGB3 & -1.73 & 0 \\
\hline WISP2 & -2.57 & 0 & EDIL3 & -2.24 & 0 \\
\hline THY1 & -3.58 & 4.05E-05 & CNTN1 & -2.25 & 0 \\
\hline ITGBL1 & -3.73 & $1.93 E-172$ & COL4A6 & -2.23 & $2.09 \mathrm{E}-141$ \\
\hline WNT5A & -1.99 & 5.11E-64 & CLDN16 & -1.54 & 1.99E-05 \\
\hline CLDN4 & -1.04 & $1.90 \mathrm{E}-13$ & & & \\
\hline
\end{tabular}

type chemical reactions. Although cadmium is not a direct mutagenic inducer, proposed mechanisms of cadmiuminduced carcinogenesis include formation of ROS, alteration of antioxidant enzymes, inhibition of DNA repair enzymes, and an imbalance between pro- and anti-apoptotic proteins. However, based on our sequencing data, we did not detect obvious alteration of ROS-stimulated signaling, apoptotic signaling, or DNA repair signaling. Thus, we believe that with respect to 
A

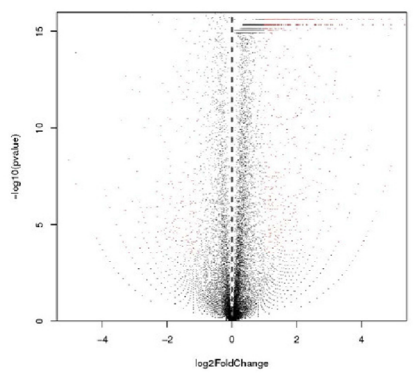

B

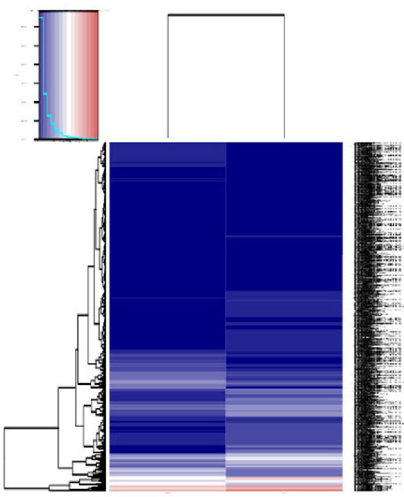

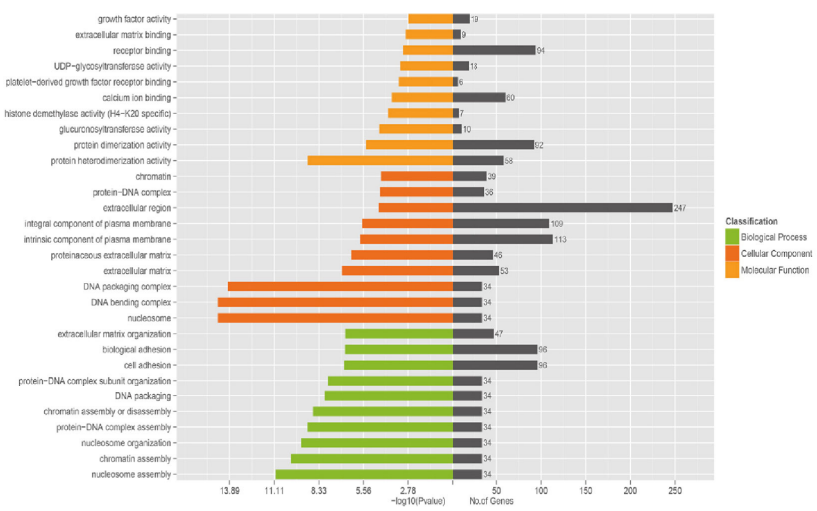

D

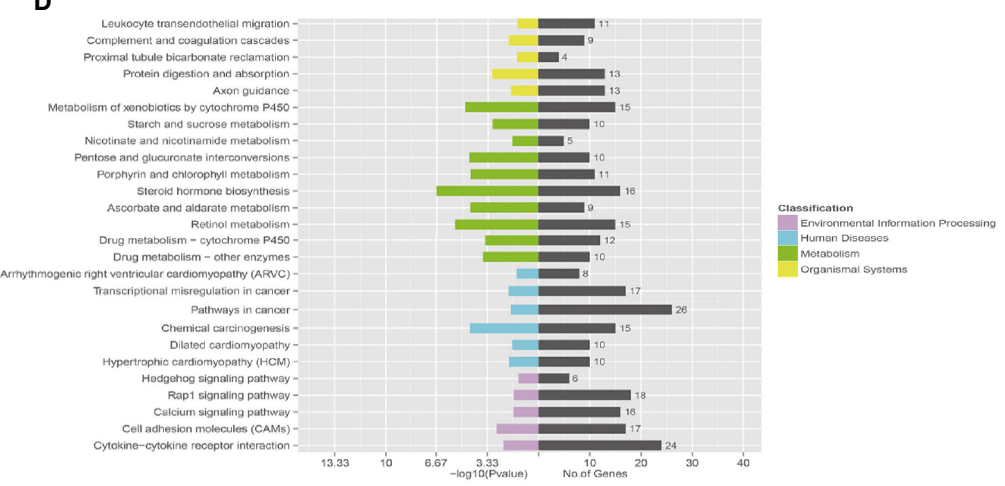

FIGURE 6 | Functional enrichment of different expression genes. (A) Volcano map of gene expression analysis between A549+Cd cells and A549+H0 cells. (B) Heat map of gene expression analysis. (C) The Gene Ontology (GO) enrichment of differentially expressed genes. GO analysis contains three branches: cellular components, molecular functions, and biological processes. (D) The Kyoto Encyclopedia of Genes and Genomes (KEGG) pathway analysis of differentially expressed genes. KEGG analysis includes four branches: environmental information processing, human diseases, metabolisms, and organismal systems.

chronic low dose cadmium exposure, ROS may not play an important role in the malignant progression of cancer. Although ROS might not be involved, we found that metabolic pathways played a critical role in Cd-induced lung cancer progression.

Several studies have identified extensive differences in metabolic profiles between cancerous and normal lung tissue $(31,32)$, which are consistent with our observations. Alterations in various metabolic pathways conferred selective advantages to cancer cells, such as producing energy and substrates for biosynthesis, increasing redox imbalance, and promoting progression of cancer (e.g., growth, proliferation, and migration) (33). Metabolic toxicity of Cd was rarely discussed in previous studies, because only a few studies indicated that $\mathrm{Cd}$ disrupted multiple metabolic pathways in adipocytes and progenitor cells (34).

Several limitations of this study are acknowledged here. First, $\mathrm{Cd}$-induced progression of adenocarcinoma was investigated in A549 cells, which might be different from that measured in lung cancer tissues. Although most of the target genes screened in our cell experiments were validated in a lung cancer cohort, applying the results from surrogate tissue to humans should be done with caution and more advanced models or human tissues are needed to confirm these alterations in the responding genome and transcriptomes. Second, the Cd dose used in this study might not represent the ordinary exposure of human lung tissue. It is difficult to determine the appropriate dose because the reported cadmium levels in lung tissue were largely inconsistent (35-37). To solve this issue, we carried out preliminary experiments and determined suitable conditions that affected the biological behavior of cells significantly without decreasing cell viability. These conditions well mimicked the conditions of chronic Cd exposure and accumulation in lung tissue. Besides, freedom from in vivo environmental pressure, especially the tumor surveillance mechanism that removes, morbid cells, may also bias the results. Finally, we only carried out experiments using A549 cells, which acted as surrogate tissue for KRAS mutant/EGFR wild type lung carcinoma. Because the A549 cell line is a KRAS-mutated lung cancer cell line already, the variants may have generated spontaneously from the in vitro tissue culture. We did not analyze the changes between the long term cultured A549 and the baseline cell or the low dose exposed A549 cells, which may undermine the impact of long-term culture to the cell line. To better understand the evolution of genomic, transcriptomic and behavioral events at different time points, the short-term, -high dose cadmium exposure model should be included in future studies. Given that lung cancer is highly heterogenous, various 
lung cancer cell lines or tissues should be utilized to explore the association between Cd-induced, genomic-/transcriptomicalterations and lung cancer more thoroughly.

In conclusion, $\mathrm{Cd}$ as a heavy toxic mental from polluted air or cigarette smoke can target human lungs directly and lead to progression of adenocarcinoma. To investigate physiological influence of lung cancer under long-term Cd exposure systematically, we established a chronic model by using A459 cells and provided a global view of genomic-/transcriptomicregulations, which linked with our morphological observations (i.e., the cancer migration and invasion). Thus, our studies based on a long-term $\mathrm{Cd}$ exposure model not only deepened our understanding of $\mathrm{Cd}$ influence on adenocarcinoma, but also could be a useful indication for future therapeutic development.

\section{MATERIALS AND METHODS}

\section{Cell Culture and Treatment}

Lung adenocarcinoma A549 cells were purchased from Cell Bank of Type Culture Collection of Chinese Academy of Sciences (Shanghai, China). A549 cells were cultured in DMEM medium (Invitrogen, Carlsbad, CA) that contained $10 \%$ fetal bovine serum, $100 \mathrm{U} / \mathrm{ml}$ penicillin, and $100 \mathrm{mg} / \mathrm{ml}$ streptomycin (Invitrogen). Cells were authenticated by short tandem repeat (STR) testing and amelogenin to the reference profile of A549 (ATCC CCL-185) (Supplementary Figure 1).

For cadmium exposures, cadmium chloride hemipentahydrate (Acros Organics, Gael, Belgium) was added to the media and applied evenly to the cultured cells. Cadmium chloride hemipentahydrate (Acros Organics, Gael, Belgium) at $1 \mu \mathrm{M}$ was added to the culturing cells continually for 8 months to build the chronic low-dose Cd model.

\section{Examination of Cell Ultrastructure}

Ultrastructure of A549+Cd cells and A549+H0 cells were observed using a transmission electron microscope (TEM). Cells were fixed with $2.5 \%$ glutaraldehyde (v/v) in $100 \mathrm{mM}$ phosphate buffer (PBS, pH 7.0) for $2 \mathrm{~h}$. Cells were washed three times with PBS and post-fixed in $1 \%$ osmium tetroxide $\left(\mathrm{OsO}_{4}\right)$ for $1 \mathrm{~h}$. Cells were dehydrated with an ethanol series, infiltrated, embedded in araldite and sectioned at $70 \mathrm{~nm}$ thickness using an ultramicrotome. Ultrathin sections were stained with $2 \%$ uranyl acetate and $0.2 \%$ lead citrate. Sections were examined using a TEM (JEOL JEM-1230 EX, Japan) at $80 \mathrm{Kv}$, and the images were observed.

\section{Immunofluorescent Staining}

All fluorescent trackers were obtained from Beyotime Biotechnology (Shanghai, China). These trackers were used to stain responding intracellular proteins or organelles, which included cell actin (Actin-Tracker Green, at ratio of $1 / 40$ ), mitochondria (Mito-Tracker Green, at ratio of $1 / 40$ ), endoplasmic reticulum (ER-Tracker Red, at ratio of $1 / 40$ ), Golgi (Golgi-Tracker Red, at ratio of 1/40), and lysosome (Lyso-Tracker Red, at ratio of $1 / 40$ ), nucleus (DAPI, at
$1 \mu \mathrm{g} / \mathrm{mL}$ ). After being washed with PBS three times, cells were visualized under an inverted fluorescence microscope (Leica, Wetzlar, Germany).

\section{Scratch Test Migration Assay and Matrigel Transwell Invasive Assay}

A two-chambered culture insert (Ibidi, Wisconsin, US) was placed in a $35 \mathrm{~mm}$ dish. The cells $\left(2.5 \times 10^{5}\right.$ cells/chamber $)$ were then seeded into the insert for $24 \mathrm{~h}$ until fully confluent attachment. After that, the inserts were removed using sterile forceps to create an even $500 \mu \mathrm{m}$ cell-free gap. Cells were washed carefully with PBS once to remove any floating cells. Cell migration was analyzed at different time points $(0 \mathrm{~h}, 6 \mathrm{~h}, 12 \mathrm{~h}$, and $24 \mathrm{~h}$ ) by a Nikon TMS-F phase contrast microscope (Tokyo, Japan).

For the Matrigel Transwell invasive assays, A549+Cd cells and A549+H0 cells were collected and resuspended at a density of $1 \times 10^{5}$ in $100 \mathrm{ml}$ of serum-free medium and then seeded into the upper chamber (Corning, USA) with a Matrigel-coated membrane (24-well insert; 8 -mm pore size) according to the manufacturer's protocol. Afterwards, we filled the lower chambers with DMEM that contained 10\% FBS. After incubation for the indicated time, non-invasive cells on the upper surface of the upper chamber were removed mechanically by cotton swabs; the cells on the lower surface of filters were fixed with methanol for $30 \mathrm{~min}$ and stained with $0.1 \%$ crystal violet for $30 \mathrm{~min}$. The number of invasive cells was counted in five random 200 fields using an inverted microscope (Olympus IX51; Olympus America Inc., Melville, NY, USA).

\section{WES Analysis and Exome Data Analyses}

For exome sequencing, DNA of A549 cells (with/without Cd treatments) were extracted by a Qiagen genomic DNA extraction kit (Qiagen GmbH, Germany). DNA libraries were then prepared using a NEXTflexTM Rapid DNA Sequencing Kit (5144-02). The libraries were tested for enrichment by qPCR; their size distribution and concentration were determined by an Agilent Bioanalyzer 2100. After the library construction, WES was performed on an Illumina HiSeq3000 sequencer (version 3, Illumina, Inc., California, USA) in high-output mode with 150 bp paired-end reads. The consequent WES data were analyzed by a pipeline with three major steps: 1) quality control (QC) of raw data; 2) sequence read mapping; and 3) single-nucleotide polymorphism (SNP) analyses. In detail, QC was performed on the NGS raw data (FASTQ format) by using FastQC (38), which provides a thorough examination of the reads. Raw reads were cleaned by removal of adapter sequences and low-quality reads (Phred quality <20) and then applied to genome mapping. Different attributes of reads (e.g. Phred score, GC ratio, reads coverage, adapter/primer influences, and sequence duplicates) were checked, trimmed, and filtered to present high-quality reads for sequence mapping. The sequences were then mapped to the human genome (hg19) using the Burrows-Wheeler Aligner (0.7.12) (39). After mapping, GATK/Picard (40) software was employed to detect SNP loci with PHRED score $>30$ (i.e., error rate $<1 / 1000$ ). Subsequently, ANNOVAR (41) was used to 
annotate SNP loci to determine whether the detected SNPs were in the known database (i.e., dbSNP138SNP database, 1000 Genome database and ESP6500 human exon database), gene functional annotation, exonic variant annotation, and heterozygosity.

GO and KEGG analysis were performed through the DAVID (https://david.ncifcrf.gov) database (42). Besides, to evaluate further the pathogenicity of these SNPs in 78 mutated genes associated with cell migration and invasion, we checked on Pathogenicity Calculator (http://calculator.clinicalgenome.org/ site/cg-calculator) (43). To analyze the 78 mutated genes associated with cell migration and invasion, the human lung adenocarcinoma SNP mutation data and transcriptome data (normal: 54, tumor: 497) (TCGA-LUAD) were downloaded from the Cancer Genome Atlas (TCGA) database through DESeq2 installation package (44). The overlap of up-regulated mutated genes and down-regulated mutated genes were visualized using the R package "maftools".

\section{RNA Sequencing and Data Analysis}

Total RNA was extracted and purified by using Qiagen RNeasy Mini Kit (Hilden, Germany). The purity, concentration and integrity of total RNA were detected by NanoDrop 2000 spectrophotometer, Qubit 3.0 Fluorometer and Agilent 2100 Bioanalyzer, respectively. To retain all lncRNAs, which included those with or without a poly (A) tail, ribosomal RNA was removed by an Epicentre Ribo-zero ${ }^{\text {TM }}$ rRNA Removal Kit (Epicentre, USA). The purified RNA was fragmented, converted into cDNA with adenylation of 3' ends, and then amplified by using PCR. RNA-seq was then performed by Bai Hao Biological Technologies (Liaoning, China), using an Illumina HiSeq3000 platform (50 cycles with 150 base pair-end reads). Totals of $61,156,328$ and 47,872,203 pair-end reads remain in $\mathrm{A} 549+\mathrm{Cd}$ and $\mathrm{A} 549+\mathrm{H} 0$ cells, respectively, after the QC process, which included the removal of adapter sequences, contaminated sequences, and low-quality sequences (Phred quality $<20$ or $\mathrm{N}$ base $>10 \%)$. We also used the RNAcentral database (45) to remove the rRNAs that would affect the lncRNA data analysis, and $121,308,770$ and 94,864,580 reads remained for A549+Cd and $\mathrm{A} 549+\mathrm{H} 0$ cells, respectively, for the mapping process. Tophat2 (46) was used to map reads to the Ensemble GRCh37/hg19 (iGenome version) reference with the default parameters (-read-mismatches $=2$ and -read-gap-length $=2$ ). Subsequently, we obtained $75,968,484$ mapped reads that were distributed in exonic (80.9\%), intergenic(3.4\%), intronic(15.3\%) and splicing(0.4\%) in A549+Cd. A549+H0 had a similar result with $69,272,540$ mapped reads distributed in exonic (78.6\%), intergenic (4.0\%), intronic (17.0\%) and splicing $(0.4 \%)$. The unique mapped reads were subjected to subsequent processing, such as removing PCR duplicates, before counting transcripts. Differential expression analysis (Cd treatments compared with Cd non-treatments) was conducted by a standard workflow, using Audics (47) for genes, mRNA and lncRNA. The Benjamini-Hochberg multiple test correction was enabled by default during the analysis. All thresholds for significant differential expression were then set as q-value $<0.05 \& \mid \log 2$ (Fold change) $\mid>1.5$.

\section{Statistical Analysis}

The results of Scratch test migration assay and Matrigel Transwell invasive assay were expressed as mean \pm standard deviation calculated from three independent experiments. The data were analyzed with an independent samples t-test, SPSS 17.0 software. Values of $p<0.05$ were taken as statistically significant. The workflow of DEseq2 and AudicS used the BenjaminiHochberg multiple test correction (FDR) to correct $p$-value to q-value.

\section{DATA AVAILABILITY STATEMENT}

The datasets presented in this study can be found in online repositories. The names of the repository/repositories and accession number(s) can be found in the article/Supplementary Material.

\section{AUTHOR CONTRIBUTIONS}

SDD contributed to the conception of the work, designed the work and drafted the manuscript. SW participated in the acquisition and analysis of the data. JCZ and YNQ interpreted the data and revised the manuscript. All authors contributed to the article and approved the submitted version.

\section{FUNDING}

This work was supported by grants from National Natural Science Foundation of China (No. 81401881 to SDD), Natural Science Foundation of Liaoning Province (No. 2014021018 to SDD), and Fundamental research program funding of Ninth People's Hospital affiliated to Shanghai Jiao Tong university School of Medicine (No. JYZZ144 to SDD).

\section{ACKNOWLEDGMENTS}

Authors would like to thank Thomas A. Gavin, Professor Emeritus, Cornell University, for help with editing this paper.

\section{SUPPLEMENTARY MATERIAL}

The Supplementary Material for this article can be found online at: https://www.frontiersin.org/articles/10.3389/fonc.2021.654687/ full\#supplementary-material

Supplementary Figure 1 | STR profiles of parental A549 cells, A549+H0 cells and A549+Cd cells.All 30 STR loci detected in our cells were matched with the ATCC 30 STR loci of lung adenocarcinoma A549 cells, and the match degree was $100 \%$. The result confirmed the identity of cells used in our study and excluded the possibility of cross-contamination during long-term cell passage. 


\section{REFERENCES}

1. Aitio A, Alessio L, Axelson O, De Flora S, Grandjean P, Heinrich U, et al. Cadmium and Cadmium Compounds. In: IARC Monographs on the Evaluation of Carcinogenic Risks to Humans Lyon: International Agency for Research on Cancer (1993). 58 119-237 p.

2. Hsieh CY, Jung CR, Lin CY, Hwang BF. Combined Exposure to Heavy Metals in PM(2.5) and Pediatric Asthma. J Allergy Clin Immunol (2020) 147:217180.e13. doi: 10.1016/j.jaci.2020.12.634

3. Waalkes MP. Cadmium Carcinogenesis in Review. J Inorganic Biochem (2000) 79:241-4. doi: 10.1016/s0162-0134(00)00009-x

4. McMurray CT, Tainer JA. Cancer, Cadmium and Genome Integrity. Nat Genet (2003) 34:239-41. doi: 10.1038/ng0703-239

5. Cao X, Fu M, Bi R, Zheng X, Fu B, Tian S, et al. Cadmium Induced BEAS-2B Cells Apoptosis and Mitochondria Damage via MAPK Signaling Pathway. Chemosphere (2021) 263:128346. doi: 10.1016/j.chemosphere.2020.128346

6. Tanwar VS, Zhang X, Jagannathan L, Jose CC. S. Cuddapah. Cadmium Exposure Upregulates SNAIL Through miR-30 Repression in Human Lung Epithelial Cells. Toxicol Appl Pharmacol (2019) 373:1-9. doi: 10.1016/ j.taap.2019.04.011

7. Galán A, García-Bermejo L, Troyano A, Vilaboa NE, Fernández C, de Blas E, et al. The Role of Intracellular Oxidation in Death Induction (Apoptosis and Necrosis) in Human Promonocytic Cells Treated With Stress Inducers (Cadmium, Heat, X-Rays). Eur J Cell Biol (2001) 80:312-20. doi: 10.1078/0171-9335-00159

8. Bade BC, Dela Cruz CS. Lung Cancer 2020: Epidemiology, Etiology, and Prevention. Clinics Chest Med (2020) 41:1-24. doi: 10.1016/j.ccm.2019.10.001

9. Waalkes MP. Cadmium Carcinogenesis. Mutat Res (2003) 533:107-20. doi: 10.1016/j.mrfmmm.2003.07.011

10. Luo H, Li Z, Ge H, Mei D, Zhao L, Jiang L, et al. HMGA2 Upregulation Mediates Cd-Induced Migration and Invasion in A549 Cells and in Lung Tissues of Mice. Chemico Biological Interactions (2017) 277:1-7. doi: 10.1016/ j.cbi.2017.08.012

11. Liu F, Wang B, Li L, Dong F, Chen X, Li Y, et al. Low-Dose Cadmium Upregulates VEGF Expression in Lung Adenocarcinoma Cells. Int J Environ Res Public Health (2015) 12:10508-21. doi: 10.3390/ijerph120910508

12. Jumarie C. Cadmium Transport Through Type II Alveolar Cell Monolayers: Contribution of Transcellular and Paracellular Pathways in the Rat ATII and the Human A549 Cells. Biochim Biophys Acta (2002) 1564:487-99. doi: 10.1016/s0005-2736(02)00499-6

13. Luparello C, Sirchia R, Longo A. Cadmium as a Transcriptional Modulator in Human Cells. Crit Rev Toxicol (2011) 41:75-82. doi: 10.3109/10408444. 2010.529104

14. Jose CC, Jagannathan L, Tanwar VS, Zhang X, Zang C, Cuddapah S. Nickel Exposure Induces Persistent Mesenchymal Phenotype in Human Lung Epithelial Cells Through Epigenetic Activation of ZEB1. Mol Carcinog (2018) 57:794-806. doi: 10.1002/mc.22802

15. Krämer A, Green J, Pollard JJr., Tugendreich S. Causal Analysis Approaches in Ingenuity Pathway Analysis. Bioinf (Oxford England) (2014) 30:523-30. doi: 10.1093/bioinformatics/btt703

16. Masters JR, Thomson JA, Daly-Burns B, Reid YA, Dirks WG, Packer P, et al. Short Tandem Repeat Profiling Provides an International Reference Standard for Human Cell Lines. Proc Natl Acad Sci USA (2001) 98:8012-7. doi: 10.1073/ pnas. 121616198

17. Calabrese EJ, Stanek EJ3rd, Nascarella MA, Hoffmann GR. Hormesis Predicts Low-Dose Responses Better Than Threshold Models. Int J Toxicol (2008) 27:369-78. doi: 10.1080/10915810802503735

18. Kundu S, Sengupta S, Bhattacharyya A. EGFR Upregulates Inflammatory and Proliferative Responses in Human Lung Adenocarcinoma Cell Line (A549), Induced by Lower Dose of Cadmium Chloride. Inhalation Toxicol (2011) 23:339-48. doi: 10.3109/08958378.2011.572931

19. Li B, Xiao R, Wang C, Cao L, Zhang Y, Zheng S, et al. Spatial Distribution of Soil Cadmium and Its Influencing Factors in Peri-Urban Farmland: A Case Study in the Jingyang District, Sichuan, China. Environ Monit Assessment (2017) 189:21. doi: 10.1007/s10661-016-5744-y

20. Nawrot TS, Staessen JA, Roels HA, Munters E, Cuypers A, Richart T, et al. Cadmium Exposure in the Population: From Health Risks to Strategies of Prevention. Biometals an Int J Role Metal Ions Biol Biochem Med (2010) 23:769-82. doi: 10.1007/s10534-010-9343-z
21. Casalino E, Sblano C, Landriscina C. Enzyme Activity Alteration by Cadmium Administration to Rats: The Possibility of Iron Involvement in Lipid Peroxidation. Arch Biochem Biophys (1997) 346:171-9. doi: 10.1006/abbi.1997.0197

22. Yuan W, Yang N, Li X. Advances in Understanding How Heavy Metal Pollution Triggers Gastric Cancer. BioMed Res Int (2016) 2016:7825432. doi: $10.1155 / 2016 / 7825432$

23. Knani L, Venditti M, Kechiche S, Banni M, Messaoudi I, Minucci S. Melatonin Protects Bone Against Cadmium-Induced Toxicity via Activation of Wnt $/ \beta$ Catenin Signaling Pathway. Toxicol Mech Methods (2020) 30:237-45. doi: 10.1080/15376516.2019.1701595

24. Peng L, Huang YT, Zhang F, Chen JY, Huo X. Chronic Cadmium Exposure Aggravates Malignant Phenotypes of Nasopharyngeal Carcinoma by Activating the Wnt/ $\beta$-Catenin Signaling Pathway via Hypermethylation of the Casein Kinase $1 \alpha$ Promoter. Cancer Manage Res (2019) 11:81-93. doi: $10.2147 / \mathrm{cmar} . S 171200$

25. Zhang C, Lin T, Nie G, Hu R, Pi S, Wei Z, et al. Cadmium and Molybdenum Co-Induce Pyroptosis via ROS/PTEN/PI3K/AKT Axis in Duck Renal Tubular Epithelial Cells. Environ Pollut (Barking Essex 1987) (2020) 272:116403. doi: 10.1016/j.envpol.2020.116403

26. Kulkarni P, Dasgupta P, Bhat NS, Hashimoto Y, Saini S, Shahryari V, et al. Role of the PI3K/Akt Pathway in Cadmium Induced Malignant Transformation of Normal Prostate Epithelial Cells. Toxicol Appl Pharmacol (2020) 409:115308. doi: 10.1016/j.taap.2020.115308

27. Huang CC, Kuo CY, Yang CY, Liu JM, Hsu RJ, Lee KI, et al. Cadmium Exposure Induces Pancreatic $\beta$-Cell Death via a Ca(2+)-Triggered JNK/ CHOP-Related Apoptotic Signaling Pathway. Toxicology (2019) 425:152252. doi: 10.1016/j.tox.2019.152252

28. Son YO, Wang L, Poyil P, Budhraja A, Hitron JA, Zhang Z, et al. Cadmium Induces Carcinogenesis in BEAS-2B Cells Through ROS-Dependent Activation of PI3K/AKT/GSK-3 $\beta / \beta$-Catenin Signaling. Toxicol Appl Pharmacol (2012) 264:153-60. doi: 10.1016/j.taap.2012.07.028

29. Genchi G, Sinicropi MS, Lauria G, Carocci A, Catalano A. The Effects of Cadmium Toxicity. Int J Environ Res Public Health (2020) 17:3782. doi: $10.3390 /$ ijerph 17113782

30. Fu Z, Xi S. The Effects of Heavy Metals on Human Metabolism. Toxicol Mech Methods (2020) 30:167-76. doi: 10.1080/15376516.2019.1701594

31. Tang Y, Li Z, Lazar L, Fang Z, Tang C, Zhao J. Metabolomics Workflow for Lung Cancer: Discovery of Biomarkers. Clinica Chimica Acta; Int J Clin Chem (2019) 495:436-45. doi: 10.1016/j.cca.2019.05.012

32. Weinberg F, Dickson RP, Nagrath D, Ramnath N. The Lung Microbiome: A Central Mediator of Host Inflammation and Metabolism in Lung Cancer Patients? Cancers (2020) 13:13. doi: 10.3390/cancers 13010013

33. Dias AS, Almeida CR, Helguero LA, Duarte IF. Metabolic Crosstalk in the Breast Cancer Microenvironment. Eur J Cancer (Oxford Engl 1990) (2019) 121:154-71. doi: 10.1016/j.ejca.2019.09.002

34. Jablonska E, Socha K, Reszka E, Wieczorek E, Skokowski J, Kalinowski L, et al. Cadmium, Arsenic, Selenium and Iron- Implications for Tumor Progression in Breast Cancer. Environ Toxicol Pharmacol (2017) 53:151-7. doi: 10.1016/ j.etap.2017.05.014

35. Pinto E, Cruz M, Ramos P, Santos A, Almeida A. Metals Transfer From Tobacco to Cigarette Smoke: Evidences in Smokers' Lung Tissue. J Hazardous Mater (2017) 325:31-5. doi: 10.1016/j.jhazmat.2016.11.069

36. Fortoul TI, Osorio LS, Tovar AT, Salazar D, Castilla ME, Olaiz-Fernández G. Metals in Lung Tissue From Autopsy Cases in Mexico City Residents: Comparison of Cases From the 1950s and the 1980s. Environ Health Perspect (1996) 104:630-2. doi: 10.1289/ehp.96104630

37. Kollmeier H, Seemann J, Wittig P, Rothe G, Müller KM. Cadmium in Human Lung Tissue. Int Arch Occup Environ Health (1990) 62:373-7. doi: 10.1007/ bf00381367

38. Andrews S. Babraham Bioinformatics (2010) Available at: http://www. bioinformatics.babraham.ac.uk/projects/fastqc [Accessed November 4, 2021].

39. Li H, Durbin R. Fast and Accurate Short Read Alignment With BurrowsWheeler Transform. Bioinformatics (2009) 25:1754-60. doi: 10.1093/ bioinformatics/btp324

40. McKenna A, Hanna M, Banks E, Sivachenko A, Cibulskis K, Kernytsky A, et al. The Genome Analysis Toolkit: A MapReduce Framework for Analyzing Next-Generation DNA Sequencing Data. Genome Res (2010) 20:1297-303. doi: $10.1101 /$ gr. 107524.110 
41. Wang K, Li M, Hakonarson H. ANNOVAR: Functional Annotation of Genetic Variants From High-Throughput Sequencing Data. Nucleic Acids Res (2010) 38:e164. doi: 10.1093/nar/gkq603

42. Huang da W, Sherman BT, Lempicki RA. Systematic and Integrative Analysis of Large Gene Lists Using DAVID Bioinformatics Resources. Nat Protoc (2009) 4:44-57. doi: 10.1038/nprot.2008.211

43. Richards S, Aziz N, Bale S, Bick D, Das S, Gastier-Foster J, et al. Standards and Guidelines for the Interpretation of Sequence Variants: A Joint Consensus Recommendation of the American College of Medical Genetics and Genomics and the Association for Molecular Pathology. Genet Med (2015) 17:405-24. doi: 10.1038/gim.2015.30

44. Love MI, Huber W, Anders S. Moderated Estimation of Fold Change and Dispersion for RNA-Seq Data With Deseq2. Genome Biol (2014) 15:550. doi: 10.1186/s13059-014-0550-8

45. The RC, Petrov AI, Kay SJE, Kalvari I, Howe KL, Gray KA, et al. RNAcentral: A Comprehensive Database of Non-Coding RNA Sequences. Nucleic Acids Res (2017) 45:D128-34. doi: 10.1093/nar/gkw1008

46. Kim D, Pertea G, Trapnell C, Pimentel H, Kelley R, Salzberg SL. TopHat2: Accurate Alignment of Transcriptomes in the Presence of Insertions, Deletions and Gene Fusions. Genome Biol (2013) 14:R36. doi: 10.1186/gb-2013-14-4-r36
47. Audic S, Claverie JM. The Significance of Digital Gene Expression Profiles. Genome Res (1997) 7:986-95. doi: 10.1101/gr.7.10.986

Conflict of Interest: The authors declare that the research was conducted in the absence of any commercial or financial relationships that could be construed as a potential conflict of interest.

Publisher's Note: All claims expressed in this article are solely those of the authors and do not necessarily represent those of their affiliated organizations, or those of the publisher, the editors and the reviewers. Any product that may be evaluated in this article, or claim that may be made by its manufacturer, is not guaranteed or endorsed by the publisher.

Copyright (๑) 2021 Dai, Wang, Qin and Zhu. This is an open-access article distributed under the terms of the Creative Commons Attribution License (CC BY). The use, distribution or reproduction in other forums is permitted, provided the original author(s) and the copyright owner(s) are credited and that the original publication in this journal is cited, in accordance with accepted academic practice. No use, distribution or reproduction is permitted which does not comply with these terms. 